\title{
Integrated outpatient treatment increased abstinence in men with alcohol related illness who were ongoing drinkers
}

Willenbring $M L$, Olson DH. A randomized trial of integrated outpatient treatment for medically ill alcoholic men. Arch Intern Med 1999 Sep 13;159:1946-52.

QUESTION: In men with alcohol related illness who were ongoing drinkers, does integrated outpatient treatment (IOT) increase abstinence, remission of alcohol dependence, quality of life, and survival?

\section{Design}

Randomised (allocation concealed*), unblinded,* controlled trial with 2 year follow up.

Setting

Veterans Affairs Medical Center, Minneapolis, Minnesota, USA.

\section{Patients}

105 men (mean age 55 y) who had severe alcohol related medical illness and had been drinking heavily in the previous 6 months. Exclusion criteria were inability or unwillingness to attend monthly visits, non-alcohol related illness with poor prognosis, severe dementia, major psychiatric disorder other than depression, current substance abuse other than alcohol, or civil commitment to treatment or pending commitment. Follow up was $96 \%$.

\section{Intervention}

Men were allocated to IOT $(\mathrm{n}=48)$ or standard care $(n=53)$. IOT consisted of an inpatient evaluation by a multidisciplinary team; a treatment plan including specific goals negotiated with the patient; monthly primary care visits to measure biological indicators of heavy drinking and offer encouragement and problem solving help; and efforts to involve family members.

Source of funding: Department of Veterans Affairs Research Service, Washington,

$D C$.

For correspondence: Dr M L Willenbring,

Psychiatry Service

(116A), VA Medical

Center, One Veterans

Drive, $M N$

55417-2300, USA

Fax +1612970

5891.Email

vhaminwillem@

med.va.gov.

Abstract and

commentary also

published in ACP

Journal Club.

\section{Main outcome measures}

Drinking in previous 30 days, alcohol dependence symptoms in previous year, Addiction Severity Index (ASI) scores, medical service use, quality of life (Treatment of Mild Hypertension Study Health and Well-being Scale scores), and death at 2 years.

\section{Main results}

More men in the IOT group than the control group reported abstinence in the previous 30 days $(p=0.02)$ (table). Men in the IOT group had more outpatient visits (mean $42 v 17$ visits, $\mathrm{p}<0.01$ ) than did men in the control group. Quantity and frequency of drinking among men who continued to drink did not differ between groups. ASI psychiatric ratings were higher in the IOT group than in the control group at baseline and follow up. The groups did not differ for mean alcohol depend-

Integrated outpatient treatment (IOT) v standard care (control) for alcohol related illness and ongoing drinking at 2 years $\dagger$

\begin{tabular}{lllll} 
Outcomes & IOT & Standard care & RBI (95\% CI) & NNT (CI) \\
Survival & $81 \%$ & $70 \%$ & $16 \%(-7$ to 48$)$ & Not significant \\
\hline Abstinence in previous 30 d & $74 \%$ & $49 \%$ & $51 \%(5$ to 128$)$ & $4(3$ to 35$)$ \\
\hline
\end{tabular}

†Abbreviations defined in glossary; $\mathrm{RBI}, \mathrm{NNT}$, and $\mathrm{Cl}$ calculated from data in article. ence symptoms, quality of life, ASI scores, use of hospital services, or survival at follow up (table) except where differences existed at baseline.

\section{Conclusion}

In men with alcohol related medical conditions who were ongoing drinkers, integrated outpatient treatment was associated with greater self reported abstinence and use of outpatient services than was standard care.

*See glossary.

\section{COMMENTARY}

This provocative study by Willenbring and Olson is the first randomised controlled trial of a longitudinal, primary care intervention in alcohol dependent patients who have serious alcohol related medical conditions. A larger trial, with blinded assessment of outcomes in interviews, is needed to unequivocally show the benefit of this approach. However, this study is important for several reasons. Firstly, the intervention was acceptable to patients. Patients who were unlikely to accept referral for specialised alcohol treatment were willing to see primary care providers monthly. Secondly, the programme did not require patients to accept a goal of abstinence but nevertheless resulted in increased self reported abstinence. Third, the intervention focused partly on tracking biologic markers of disease, which is done routinely in the management of other chronic conditions and is likely to make the intervention acceptable to primary care providers.

Future studies will need to evaluate specific components of the multifaceted intervention. Potential active ingredients include negotiation of treatment goals with patients, ${ }^{1}$ standardised progress notes that may act as prompts to address the patient's drinking at each visit, repeated review of objective biologic indicators of harmful drinking, ${ }^{2}$ and use of protocols and consultants to support primary care providers. ${ }^{3}$

Until future study results are available, providers who work with actively drinking patients with alcohol related medical conditions should consider scheduling frequent visits specifically to address patients' drinking, identify and provide repeated feedback to patients regarding any improvement or deterioration in objective measures of alcohol related harm, and offer referrals as needed to support achievement of abstinence. Katharine A Bradley, MD University of Washington Seattle, Washington, USA

1 Williams GC, Quill TE, Deci EL, et al. "The facts concerning the recent carnival of smoking in Connecticut" and elsewhere. Ann Intern Med 1991:115:59-63.

2 Kristenson $\mathrm{H}$, Ohlin $\mathrm{H}$, Hulten-Nosslin MB, et al. Identification and intervention of heavy drinking in middle-aged men: results and follow-up of 24-60 months of long-term study with randomized controls. Alcohol Clin Exp Res 1983;7:203-9.

3 Katon W, Von Korff M, Lin E, et al. Collaborative management to achieve treatment guidelines. Impact on depression in primary care. JAMA 1995;273:1026-31. 